\title{
Viewpoint
}

\section{For faster magnetic switching-destroy and rebuild}

\author{
Riccardo Hertel \\ Institute of Solid State Research, IFF-9, Forschungszentrum Jülich GmbH, D-52425 Jülich, Germany
}

Published September 8, 2009

Magnetic switching is typically a continuous process, where a field pulse rotates a magnet from up to down, but it is now possible to do this faster — and with all-optical methods - by first quenching the magnetization to zero and then repolarizing it in the opposite direction.

Subject Areas: Optics, Magnetism

A Viewpoint on:

Ultrafast Path for Optical Magnetization Reversal via a Strongly Nonequilibrium State

K. Vahaplar, A. M. Kalashnikova, A. V. Kimel, D. Hinzke, U. Nowak, R. Chantrell, A. Tsukamoto, A. Itoh, A. Kirilyuk and Th. Rasing

Phys. Rev. Lett. 103, 117201 (2009) - Published September 8, 2009

Magnetic data storage technology and the everincreasing speed of information processing have brought enormous changes to our daily life. These developments naturally lead us to ask if there is a physical limit to the speed at which magnetic moments can be switched [1] - a topic that has caused no shortage of controversy in the scientific community. Exploring this limit is complicated, partly because switching the magnetization from one direction to the other can occur in multiple ways and along different paths. For example, magnetic and electric fields, electric currents, and laser pulses can all stimulate magnetic switching and the trajectory of the magnetization vector from its initial to its final state will vary with each of these switching mechanisms.

Kadir Vahaplar and colleagues at Radboud University Nijmegen in The Netherlands, in collaboration with scientists in Germany, the UK, Japan, and Russia have made a dramatic leap forward in exploring the limits to magnetic switching. Writing in Physical Review Letters, they demonstrate a magnetic write-read event that occurs on times as short as 30 picoseconds (ps), which is the fastest magnetic switching process observed so far [2]. But the work by Vahaplar et al. is much more than the demonstration of high-speed magnetic switching. By combining sophisticated experimental methods with theoretical tools that fully account for the magnetization on many length scales (from the continuum to the atomic and electronic limit), their study leads to important insight and detailed understanding of what fundamental processes allow ultrafast magnetic switching to occur.

So far, groups have mainly looked at ways of turning and redirecting the magnetization continuously, typically by causing it to precess with magnetic field pulses [3]. Using purely optical methods, Vahaplar et al. show

DOI: $10.1103 /$ Physics 2.73

URL: http://link.aps.org/doi/10.1103/Physics.2.73 that a faster way to switch the magnetization is to temporarily quench it [4], that is, reduce it to zero, and restore it immediately afterwards in the opposite direction, a scheme they aptly call a linear reversal (Fig. 1).

Their experiments are an ingenious combination of the different effects by which light interacts with magnetic moments. These effects are usually categorized as optomagnetic or magneto-optical, depending on whether they describe the influence of the light pulse on the magnetization or vice versa. In their setup, Vahaplar et al. first stimulate the magnetization of amorphous $20 \mathrm{~nm}$ ferromagnetic films made of $\mathrm{Gd}_{x} \mathrm{Fe}_{100-x-y} \mathrm{Co}_{y}$ with a short and intense circularly polarized (pump) laser pulse and then image the magnetization with a second, equally short but linearly polarized (probe) laser pulse.

The first laser pulse has two effects on the magnetization. First, it rapidly pumps energy into the film, locally heating the material and demagnetizing it [5]. The energy of the laser pulse is primarily absorbed by the electrons, which reach a temperature of about $1200 \mathrm{~K}$ within the first few hundred femtoseconds (fs) after the pulse. Changes in the electronic temperature affect the magnetic properties on sub-ps time scales. Most importantly, the magnitude of the magnetization $M$ decreases as the temperature of the electronic system approaches the Curie temperature $T_{C}$ (the temperature at which the material undergoes a phase transition from a ferromagnet to a paramagnet, at equilibrium). Vahaplar et al. show that the magnetization can in fact be temporarily "destroyed" down to a value of zero about 500 fs after applying a sufficiently strong laser pulse.

The first laser pulse also affects the magnetization via the inverse Faraday effect [6]: as the circularly polarized electromagnetic field pulse traverses the sample, it acts as an effective magnetic field along the pulse's propa-

(C) 2009 American Physical Society 

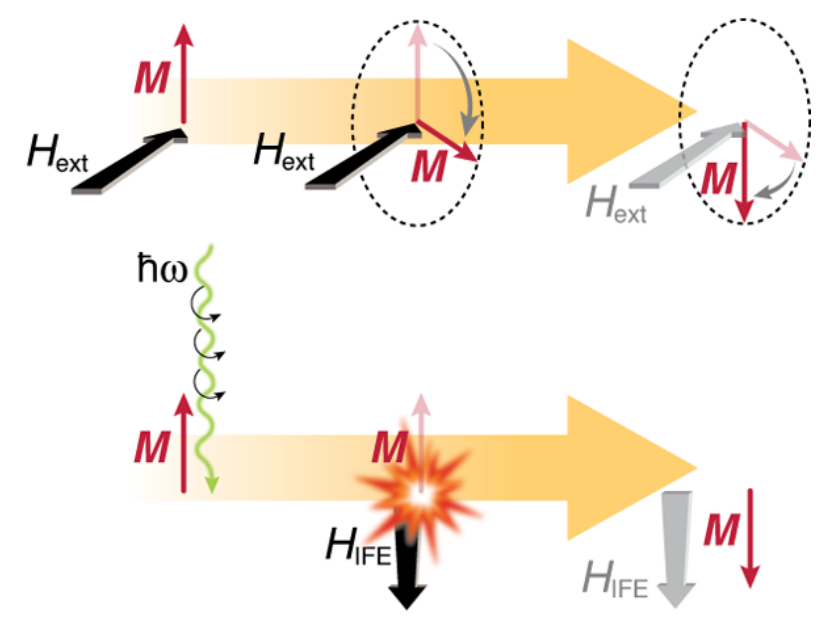

FIG. 1: A fast way of reversing the magnetization $M$ is precessional switching (top), where a short field pulse $H_{\text {ext }}$ is applied perpendicular to the magnetization. If the pulse is properly shaped, it can rotate the magnetization by $180^{\circ}$. The faster "linear" switching mode described by Vahaplar et al. (bottom) is driven by a short and intense circularly polarized laser pulse. Shortly after the pulse, the magnetization collapses due to heating. During the cooling, $M$ restores along the effective magnetic field $H_{\text {IFE }}$ that is generated by the inverse Faraday effect. (Illustration: Carin Cain)

gation direction. This effective magnetic field is proportional to the intensity of the laser pulse and to its degree of circular polarization. The inverse Faraday effect provides outstanding possibilities to control the magnetization, since it can generate locally enormously strong effective magnetic fields of up to about $20 \mathrm{~T}$. It can switch the magnetization as well, since the sign of the field only depends on the pulse's chirality. This optomagnetic, nonthermal control of the magnetization was first demonstrated by the Nijmegen group in 2007 [7]. Essentially, they showed that laser pulses as short as $40 \mathrm{fs}$ could induce optomagnetic switching, but it was not clear how much time the magnetization required to complete the switching process after the exposure to such a short pulse.

This information is a central result of the current work by Vahaplar et al. They used the well-known Faraday effect-where the magnetization of a material rotates the polarization of light transmitted through it- to image the magnetization dynamics within a few tens of picoseconds after the pump pulse. By carefully varying the delay between the circularly polarized pump pulse and the linearly polarized probe pulse, the authors could obtain precise information on the spatiotemporal evolution of the magnetization in the film. They found that the switching process completes within a time well below 90 ps, which is very short but still much longer than the duration of the pulse. The reversal is initiated in a small region in which the heating essentially destroys the magnetization for an instant. Subsequently, after about $30 \mathrm{ps}$, the magnetization nucle- ates and rebuilds as the temperature of the electrons decreases. This either leads to an expansion of the nucleus to form a larger area with a reversed magnetization or to the restoration of the initial state, depending on the initial magnetization direction and the chirality of the laser pulse.

Reliable switching only occurs within a narrow range of parameters for the laser pulse. The switching probability also depends critically on how high the electrons are heated: too little, and the initial magnetization will not be destroyed, too much and the material will not cool down quickly enough to restore the magnetization in the opposite direction before all information is lost. Vahaplar et al. performed sophisticated simulation studies to build a phase diagram of the suitable combinations of laser pulse duration and intensity, which are confirmed by the experiments.

The technique of locally heating the magnetization above the Curie temperature and restoring it in the opposite direction by applying a magnetic field upon cooling is well known in the magnetic recording industry [8]. A recent application of this concept is heat-assisted magnetic recording in high-density magnetic data storage, where a heating laser is combined with an inductive write head that provides the field along which the magnetization rebuilds as the temperature drops. This idea is also similar to the writing process in magnetooptical drives, a technology that has been popular since the nineties. In both of these cases, however, the desire to increase the storage density more so than increasing the writing speed was the main drive behind the technology.

What has particular aesthetic appeal in the process found by Vahaplar and co-workers is that a single laser pulse does all the work: It provides both the heating via energy transfer and the switching field via the inverse Faraday effect. Since the strength of these effects depends on the intensity and duration of the irradiation, it is clear that the pulse must be properly shaped to achieve the desired effect. Remarkably, the switching appears to occur even if for a short time both the magnetization and the effective field vanish, suggesting that the system partly stores information on the previous state.

Passing through a state of quenched magnetization seems to be the key to obtain ultimate magnetic switching speed. Once the system is in a quenched state, the strong magnetic exchange interaction between electrons will rapidly restore ferromagnetism. Harnessing electron exchange-the strongest force in magnetism-is certainly a very promising way to achieve ultrafast switching. It is, for example, an essential aspect of another switching mechanism that simulations predict should occur on the time scale of a few tens of ps: magnetic vortex core switching [9]. In this case, it is the small perpendicularly magnetized region at the core of a spiralling magnetization (vortex) that is switched by a short magnetic field pulse. Although vortex core switch- 
ing is very different than the linear switching Vahaplar et al. explore, it is remarkable that both high-speed methods involve the temporary formation of a region with vanishing magnetization [10].

An important remaining question in the work from Vahaplar et al. concerns the transfer and the balance of angular momentum. The magnetization reversal is connected with a change of angular momentum, which must be provided from somewhere. Yet, it is generally agreed that the apparently simple assumption of a direct transfer of the photon spin to the magnetic system is not the solution [11], suggesting that the atomic lattice may play an important role in angular momentum conservation.

\section{References}

[1] C. H. Back and D. Pescia, Nature 428, 808 (2004).
[2] K. Vahaplar, A. M. Kalashnikova, A. V. Kimel, D. Hinzke, U. Nowak, R. Chantrell, A. Tsukamoto, A. Itoh, A. Kirilyuk, and T. Rasing, Phys. Rev. Lett. 103, 117201 (2009).

[3] H. W. Schumacher, C. Chappert, R. C. Sousa, P. P. Freitas, and J. Miltat, Phys. Rev. Lett. 90, 017204 (2003).

[4] N. Kazantseva, D. Hinzke, R. W. Chantrell, and U. Nowak, Europhys. Lett. 86, 27006 (2009).

[5] E. Beaurepaire, J. C. Merle, A. Daunois, and J.-Y. Bigot, Phys. Rev. Lett. 76, 4250 (1996).

[6] J. P. van der Ziel, P. S. Pershan, and L. Malmstrom, Phys. Rev. Lett. 15, 190 (1965).

[7] C. D. Stanciu, F. Hansteen, A. V. Kimel, A. Tsukamoto, A. Itoh, A. Kiriklyuk, and T. Rasing, Phys. Rev. Lett. 94, 237601 (2007).

[8] J. Hohlfeld, Th. Gerrits, M. Bilderbeek, Th. Rasing, H. Awano, and N. Ohta, Phys. Rev. B 65, 012413 (2001).

[9] R. Hertel, S. Gliga, M. Fähnle, and C. M. Schneider, Phys. Rev. Lett. 98, 117201 (2007).

[10] E. Feldtkeller, Z. Angew. Phy. 19, 530 (1965).

[11] B. Koopmans, M. van Kampen, J. T. Kohlhepp, and W. J. M. de Jonge, Phys. Rev. Lett. 85, 844 (2000).

\section{About the Author}

\section{Riccardo Hertel}

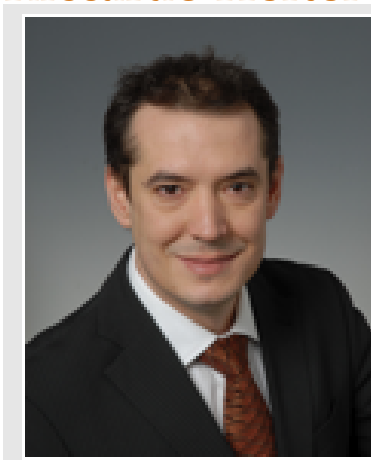

Riccardo received his Ph.D. from the University of Stuttgart, Germany, in 1999 and his Habilitation from the University of Halle-Wittenberg, Germany, in 2005. He is a lecturer at the University of Duisburg-Essen and works at the Forschungszentrum Jülich in the Institute of Solid State Research, where he is leading a research group on micro- and nanomagnetism. 